\title{
On the singular solutions of nonlinear singular partial differential equations I
}

\author{
By Hidetoshi TAHARA
}

(Received Mar. 2, 2000)

\begin{abstract}
Let us consider the following nonlinear singular partial differential equation: $\left(t \partial_{t}\right)^{m} u=F\left(t, x,\left\{\left(t \partial_{t}\right)^{j} \partial_{x}^{\alpha} u\right\}_{j+|\alpha| \leq m, j<m}\right)$ in the complex domain. Denote by $\mathscr{S}_{+}\left[\right.$resp. $\left.\mathscr{S}_{\text {log }}\right]$ the set of all the solutions $u(t, x)$ with asymptotics $u(t, x)=O\left(|t|^{a}\right)[$ resp. $\left.u(t, x)=O\left(1 /|\log t|^{a}\right)\right]$ (as $t \rightarrow 0$ uniformly in $x$ ) for some $a>0$. Clearly $\mathscr{S}_{\log } \supset \mathscr{S}_{+}$. The paper gives a sufficient condition for $\mathscr{S}_{\text {log }}=\mathscr{S}_{+}$to be valid.
\end{abstract}

The paper deals with nonlinear singular partial differential equations of the form

$$
(t \partial / \partial t)^{m} u=F\left(t, x,\left\{(t \partial / \partial t)^{j}(\partial / \partial x)^{\alpha} u\right\}_{j+|\alpha| \leq m, j<m}\right)
$$

in the complex domain. In Gérard-Tahara [1] the author has determined all the singular solutions $u(t, x)$ of (E) under the condition that $u(t, x)=O\left(|t|^{a}\right)$ (as $t \rightarrow 0$ uniformly in $x$ ) for some $a>0$.

The present paper investigates singular solutions $u(t, x)$ of (E) under a weaker condition that $u(t, x)=O\left(1 /|\log t|^{a}\right)$ (as $t \rightarrow 0$ uniformly in $x$ ) for some $a>0$.

\section{§1. Preliminaries.}

Notations: $\quad t \in \boldsymbol{C}, \quad x=\left(x_{1}, \ldots, x_{n}\right) \in \boldsymbol{C}^{n}, \quad \boldsymbol{N}=\{0,1,2, \ldots\}, \quad$ and $\quad \boldsymbol{N}^{*}=$ $\{1,2, \ldots\}$. For $\alpha=\left(\alpha_{1}, \ldots, \alpha_{n}\right) \in N^{n}$ we write $|\alpha|=\alpha_{1}+\cdots+\alpha_{n}$ and

$$
\left(\frac{\partial}{\partial x}\right)^{\alpha}=\left(\frac{\partial}{\partial x_{1}}\right)^{\alpha_{1}} \cdots\left(\frac{\partial}{\partial x_{n}}\right)^{\alpha_{n}} .
$$

Let $m \in N^{*}, N=\sharp\left\{(j, \alpha) \in \boldsymbol{N} \times \boldsymbol{N}^{n} ; j+|\alpha| \leq m, j<m\right\}$, and write the variable $Z$ as

$$
Z=\left\{Z_{j, \alpha}\right\}_{\substack{j+|\alpha| \leq m \\ j<m}} \in C^{N}
$$

2000 Mathematics Subject Classification. Primary 35A20; Secondary 35B40.

Key Words and Phrases. Nonlinear PDE, singular solutions.

This research was partially supported by Grant-in-Aid for Scientific Research (No. 12640189), The Ministry of Education, Culture, Sports, Science and Technology, Japan. 
Let $F(t, x, Z)$ be a function in the variables $(t, x, Z)$ defined in a neighborhood of the origin $(0,0,0) \in \boldsymbol{C}_{t} \times \boldsymbol{C}_{x}^{n} \times \boldsymbol{C}_{Z}^{N}$, and assume the following:

( $\left.\mathrm{A}_{1}\right) \quad F(t, x, Z)$ is holomorphic near $(0,0,0)$;

$\left(\mathrm{A}_{2}\right) \quad F(0, x, 0) \equiv 0$ near $x=0$;

(A $) \frac{\partial F}{\partial Z_{j, \alpha}}(0, x, 0) \equiv 0$ near $x=0$, if $|\alpha|>0$.

In this paper we always assume the conditions $\left(A_{1}\right),\left(A_{2}\right),\left(A_{3}\right)$, and we will consider the following nonlinear partial differential equation

$$
\left(t \frac{\partial}{\partial t}\right)^{m} u=F\left(t, x,\left\{\left(t \frac{\partial}{\partial t}\right)^{j}\left(\frac{\partial}{\partial x}\right)^{\alpha} u\right\}_{\substack{j+|\alpha| \leq m \\ j<m}}\right)
$$

with $u=u(t, x)$ as the unknown function.

For $(\mathrm{E})$ we set

$$
C(\lambda, x)=\lambda^{m}-\sum_{j<m} \frac{\partial F}{\partial Z_{j, 0}}(0, x, 0) \lambda^{j}
$$

and denote by $\lambda_{1}(x), \ldots, \lambda_{m}(x)$ the roots of the equation $C(\lambda, x)=0$ in $\lambda$. These $\lambda_{1}(x), \ldots, \lambda_{m}(x)$ are called the characteristic exponents of $(\mathrm{E})$.

The following is our basic problem:

Problem. Determine all kinds of local singularities which appear in the solutions of $(\mathrm{E})$.

Let us recall the result in Gérard-Tahara [1]. Denote:

- $\mathscr{R}(\boldsymbol{C} \backslash\{0\})$ denotes the universal covering space of $\boldsymbol{C} \backslash\{0\}$;

- $S_{\theta}=\{t \in \mathscr{R}(\boldsymbol{C} \backslash\{0\}) ;|\arg t|<\theta\}$;

- $S(\varepsilon(s))=\{t \in \mathscr{R}(\boldsymbol{C} \backslash\{0\}) ; 0<|t|<\varepsilon(\arg t)\}$, where $\varepsilon(s)$ is a positive-valued continuous function on $\boldsymbol{R}_{s}$;

- $D_{r}=\left\{x \in C^{n} ;|x| \leq r\right\}$

- $\boldsymbol{C}\{x\}$ denotes the ring of convergent power series in $x$, or equivalently the ring of germs of holomorphic functions at the origin of $\boldsymbol{C}^{n}$.

Definition 1 . We denote by $\tilde{\mathcal{O}}_{+}$the set of all $u(t, x)$ satisfying the following conditions i) and ii):

i) $u(t, x)$ is a holomorphic function on $S(\varepsilon(s)) \times D_{r}$ for some positive-valued continuous function $\varepsilon(s)$ and some $r>0$;

ii) there is an $a>0$ such that for any $\theta>0$ we have

$$
\max _{|x| \leq r}|u(t, x)|=O\left(|t|^{a}\right) \quad\left(\text { as } t \rightarrow 0 \text { in } S_{\theta}\right) .
$$


For the characteristic exponents $\lambda_{1}(x), \ldots, \lambda_{m}(x)$, we set

$$
\mu=\sharp\left\{i ; \operatorname{Re} \lambda_{i}(0)>0\right\} .
$$

When $\mu=0$, this is equivalent to the fact that $\operatorname{Re} \lambda_{i}(0) \leq 0$ for all $i=1, \ldots, m$.

When $\mu \geq 1$, by a renumeration we may assume

$$
\begin{cases}\operatorname{Re} \lambda_{i}(0)>0 & \text { for } 1 \leq i \leq \mu \\ \operatorname{Re} \lambda_{i}(0) \leq 0 & \text { for } \mu+1 \leq i \leq m\end{cases}
$$

Then we already have:

THEOREM 1 (Gérard-Tahara [1]). Denote by $\mathscr{S}_{+}$the set of all $\tilde{\mathcal{O}}_{+}$-solutions of (E). Then we have:

(I) When $\mu=0$, we have $\mathscr{S}_{+}=\left\{u_{0}\right\}$ where $u_{0}=u_{0}(t, x)$ is the unique holomorphic solution of $(\mathrm{E})$ satisfying $u_{0}(0, x) \equiv 0$.

(II) When $\mu \geq 1$, under (1.1) and the following additional conditions

1) $\lambda_{i}(0) \neq \lambda_{j}(0)$ for $1 \leq i \neq j \leq \mu$,

2) $C(1,0) \neq 0$,

3) $C\left(i+j_{1} \lambda_{1}(0)+\cdots+j_{\mu} \lambda_{\mu}(0), 0\right) \neq 0$ for any $(i, j) \in \boldsymbol{N} \times \boldsymbol{N}^{\mu}$ satisfying $i+|j| \geq 2$ (where $\left.j=\left(j_{1}, \ldots, j_{\mu}\right)\right)$,

we have

$$
\mathscr{S}_{+}=\left\{U\left(\phi_{1}, \ldots, \phi_{\mu}\right) ;\left(\phi_{1}, \ldots, \phi_{\mu}\right) \in(\boldsymbol{C}\{x\})^{\mu}\right\},
$$

where $U\left(\phi_{1}, \ldots, \phi_{\mu}\right)$ is an $\tilde{\mathcal{O}}_{+}$-solution of $(\mathrm{E})$ determined by $\left(\phi_{1}, \ldots, \phi_{\mu}\right) \in(\boldsymbol{C}\{x\})^{\mu}$ and having the expansion of the following form:

$$
\begin{aligned}
U\left(\phi_{1}, \ldots, \phi_{\mu}\right)= & \sum_{i \geq 1} u_{i}(x) t^{i} \\
& +\phi_{1}(x) t^{\lambda_{1}(x)}+\cdots+\phi_{\mu}(x) t^{\lambda_{\mu}(x)} \\
& +\sum_{\substack{i+2 m|j| \geq k+2 m \\
|j| \geq 1 \\
(i, j \mid) \neq(0,1)}} \varphi_{i, j, k}(x) t^{i+j_{1} \lambda_{1}(x)+\cdots+j_{\mu} \lambda_{\mu}(x)}(\log t)^{k} .
\end{aligned}
$$

\section{§2. Problems.}

In Theorem 1 we have restricted ourselves to the study of singular solutions in $\tilde{\mathcal{O}}_{+}$. But, there seems to be a possibility that (E) has singular solutions which do not belong in the class $\tilde{\mathcal{O}}_{+}$, as is seen in the following example.

EXAMPLE 1. The equation

$$
t \frac{\partial u}{\partial t}=u\left(\frac{\partial u}{\partial x}\right)^{k}
$$


(where $(t, x) \in C^{2}$ and $k \in N^{*}$ ) has a family of singular solutions

$$
u(t, x)=\left(\frac{1}{k}\right)^{1 / k} \frac{x+\alpha}{(c-\log t)^{1 / k}}, \quad \alpha, c \in C,
$$

which do not belong in the class $\tilde{\mathcal{O}}_{+}$.

In order to include this kind of singular solutions in our framework, we introduce the following new class of singular solutions:

Definition 2. We denote by $\tilde{\mathcal{O}}_{\text {log }}$ the set of all $u(t, x)$ satisfying the following conditions i) and ii):

i) $u(t, x)$ is a holomorphic function on $S(\varepsilon(s)) \times D_{r}$ for some positive-valued continuous function $\varepsilon(s)$ and some $r>0$;

ii) there is an $a>0$ such that for any $\theta>0$ we have

$$
\max _{|x| \leq r}|u(t, x)|=O\left(\frac{1}{|\log t|^{a}}\right) \quad\left(\text { as } t \rightarrow 0 \text { in } S_{\theta}\right) .
$$

Clearly we have $\tilde{\mathscr{O}}_{\log } \supset \tilde{\mathcal{O}}_{+}$. Therefore, if we denote by $\mathscr{S}_{\text {log }}$ the set of all

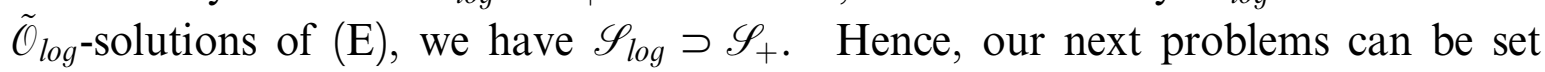
up as follows:

Problem 1. When does $\mathscr{S}_{\text {log }}=\mathscr{S}_{+}$hold?

Problem 2. When does $\mathscr{S}_{\log } \neq \mathscr{S}_{+}$hold?

The purpose of this paper is to give a partial answer and a conjecture on the problem 1. The problem 2 will be discussed in the forthcoming paper.

\section{§3. A result and a conjecture.}

In this section we will give a result on the problem 1 in a general form.

A function $\mu(t)$ on $(0, T)$ is called a weight function if it satisfies the following conditions $\left.\left.\mu_{1}\right) \sim \mu_{3}\right)$ :

$\left.\mu_{1}\right) \quad \mu(t) \in C^{0}((0, T))$

$\left.\mu_{2}\right) \mu(t)>0$ on $(0, T)$ and $\mu(t)$ is increasing in $t$,

$\left.\mu_{3}\right) \int_{0}^{T} \frac{\mu(s)}{s} d s<\infty$.

By $\mu_{2}$ ) and $\mu_{3}$ ) the condition $\mu(t) \rightarrow 0$ (as $\left.t \rightarrow+0\right)$ is clear. In this paper we impose the additional condition on $\mu(t)$ :

$$
\mu(t) \in C^{1}((0, T)) \quad \text { and } \quad\left(t \frac{d \mu}{d t}\right)(t)=o(\mu(t)) \quad(\text { as } t \rightarrow+0) .
$$

The following functions are typical examples:

$$
\mu(t)=\frac{1}{(-\log t)^{b}}, \quad \frac{1}{(-\log t)(\log (-\log t))^{c}}
$$


with $b>1, c>1$. Note that the function $\mu(t)=t^{d}$ with $d>0$ does not satisfy the condition (3.1).

DeFINITION 3. Let $\mu(t)$ be a weight function.

(1) For $a>0$ we denote by $\tilde{\mathscr{O}}_{a}(\mu(t))$ the set of all $u(t, x)$ satisfying the following conditions i) and ii):

i) $u(t, x)$ is a holomorphic function on $S(\varepsilon(s)) \times D_{r}$ for some positive-valued continuous function $\varepsilon(s)$ and some $r>0$;

ii) for any $\theta>0$ we have

$$
\max _{|x| \leq r}|u(t, x)|=O\left(\mu(|t|)^{a}\right) \quad\left(\text { as } t \rightarrow 0 \text { in } S_{\theta}\right) .
$$

(2) We define $\tilde{\mathcal{O}}_{+}(\mu(t))$ by

$$
\tilde{\mathcal{O}}_{+}(\mu(t))=\bigcup_{a>0} \tilde{\mathcal{O}}_{a}(\mu(t)) .
$$

LEMMA 1. (1) $\tilde{\mathcal{O}}_{\log }=\tilde{\mathcal{O}}_{+}(\mu(t))$ if $\mu(t)=1 /(-\log t)^{b}$ with $b>1$.

(2) If $\mu(t)$ satisfies (3.1) we have $\tilde{\mathcal{O}}_{+} \subset \tilde{\mathcal{O}}_{1}(\mu(t))\left(\subset \tilde{\mathcal{O}}_{+}(\mu(t))\right)$.

Proof. (1) is clear. (2) is verified as follows. By (3.1), for any $\varepsilon>0$ there is a $\delta>0$ such that $t \mu_{t}^{\prime}(t) \leq \varepsilon \mu(t)$ holds on $(0, \delta]$ and therefore we have

$$
\frac{d}{d t}\left(t^{-\varepsilon} \mu(t)\right) \leq 0 \text { for } 0<t \leq \delta .
$$

Integrating this from $t$ to $\delta$ we have

$$
\delta^{-\varepsilon} \mu(\delta) \leq t^{-\varepsilon} \mu(t) \text { for } 0<t \leq \delta
$$

and so

$$
\left(\frac{\mu(\delta)}{\delta^{\varepsilon}}\right) t^{\varepsilon} \leq \mu(t) \text { for } 0<t \leq \delta .
$$

Since $\varepsilon>0$ is arbitrary, (3.2) leads us to the conclusion of (2).

Denote by $\mathscr{S}_{+}(\mu(t))\left(\right.$ resp. $\left.\mathscr{S}_{a}(\mu(t))\right)$ the set of all $\tilde{\mathcal{O}}_{+}(\mu(t))$-solutions of $(\mathrm{E})$ (resp. $\tilde{\mathcal{O}}_{a}(\mu(t))$-solutions of $\left.(\mathrm{E})\right)$. By (2) of Lemma 1 we have

$$
\mathscr{S}_{+} \subset \mathscr{S}_{1}(\mu(t)) \subset \mathscr{S}_{+}(\mu(t)) .
$$

The following theorem gives a sufficient condition for $\mathscr{S}_{+}(\mu(t))=\mathscr{S}_{+}$to be valid.

THEOREM 2. Let $\mu(t)$ be a weight function satisfying (3.1). Then, $\mathscr{S}_{+}(\mu(t))$ $=\mathscr{S}_{+}$is valid if

$$
\operatorname{Re} \lambda_{i}(0)<0 \text { for all } i=1, \ldots, m
$$


or if

$$
\operatorname{Re} \lambda_{i}(0)>0 \text { for all } i=1, \ldots, m \text {. }
$$

In case (3.3), by Theorem 1 we have $\mathscr{S}_{+}=\left\{u_{0}\right\}$ and therefore the condition $\mathscr{S}_{+}(\mu(t))=\mathscr{S}_{+}$is equivalent to the fact that the local uniqueness of the solution is valid in $\mathscr{S}_{+}(\mu(t))$ which is already proved in Tahara [4], [5].

In case (3.4) the proof of Theorem 2 consists of the following two parts:

$\left.\mathrm{C}_{1}\right)$ if $u \in \mathscr{S}_{+}(\mu(t))$ we have $u \in \mathscr{S}_{m}(\mu(t))$;

$\left.\mathrm{C}_{2}\right)$ if $u \in \mathscr{S}_{m}(\mu(t))$ we have $u \in \mathscr{S}_{+}$.

The part $C_{1}$ ) will be proved in $\S 4$ and the part $C_{2}$ ) will be proved in $\S 5$.

Corollary. If (3.3) or (3.4) holds, we have $\mathscr{S}_{\text {log }}=\mathscr{S}_{+}$.

REMARK. The author believes that the following conjecture is true, though at present he has no idea to prove this conjecture:

CONJECTURE. $\mathscr{S}_{\log }=\mathscr{S}_{+}$is valid if

$$
\operatorname{Re} \lambda_{i}(0) \neq 0 \text { for all } i=1, \ldots, m \text {. }
$$

\section{§4. Proof of $\left.\mathrm{C}_{1}\right)$.}

The assertion $\mathrm{C}_{1}$ ) comes from the following proposition.

Proposition 1. Let $\mu(t)$ be a weight function satisfying (3.1). Assume the condition (3.4). Then, if $u(t, x) \in \tilde{\mathcal{O}}_{+}(\mu(t))$ is a solution of $(\mathrm{E})$ we have $u(t, x) \in \tilde{\mathcal{O}}_{m}(\mu(t))$.

\section{First we note:}

LEMMA 2. Let $\delta>0, U$ be a compact neighborhood of the origin of $\boldsymbol{C}_{x}^{n}$, $\lambda(x) \in C^{0}(U), \quad u(t, x) \in C^{1}\left((0, \delta], C^{0}(U)\right)$ and $f(t, x) \in C^{0}((0, \delta] \times U)$. Assume $\varepsilon>0, h>0, C>0, a>0$ and assume the following i) iv):

i) $t \mu_{t}^{\prime}(t) \leq \varepsilon \mu(t)$ on $(0, \delta]$,

ii) $\operatorname{Re} \lambda(x) \geq h$ on $U$,

iii) $|f(t, x)| \leq C \mu(t)^{a}$ on $(0, \delta] \times U$,

iv) $(t \partial / \partial t-\lambda(x)) u=f$ on $(0, \delta] \times U$.

Then, if a $<<$ holds we have

$$
|u(t, x)| \leq\left(\frac{|u(\delta, x)|}{\mu(\delta)^{a}}+\frac{C}{h-a \varepsilon}\right) \mu(t)^{a} \quad \text { on }(0, \delta] \times U .
$$

Proof. By solving the equation iv) we see that $u(t, x)$ is expressed by

$$
u(t, x)=\left(\frac{t}{\delta}\right)^{\lambda(x)} u(\delta, x)-\int_{t}^{\delta}\left(\frac{t}{\tau}\right)^{\lambda(x)} f(\tau, x) \frac{d \tau}{\tau}
$$


and by ii) and iii) we have

$$
|u(t, x)| \leq\left(\frac{t}{\delta}\right)^{h}|u(\delta, x)|+C \int_{t}^{\delta}\left(\frac{t}{\tau}\right)^{h} \mu(\tau)^{a} \frac{d \tau}{\tau} \quad \text { on }(0, \delta] \times U .
$$

Therefore, to show (4.1) it is sufficient to prove the following inequalities:

$$
\begin{gathered}
\left(\frac{t}{\delta}\right)^{h} \leq\left(\frac{\mu(t)}{\mu(\delta)}\right)^{a} \quad \text { on }(0, \delta], \\
\int_{t}^{\delta}\left(\frac{t}{\tau}\right)^{h} \mu(\tau)^{a} \frac{d \tau}{\tau} \leq \frac{1}{h-a \varepsilon} \mu(t)^{a} \quad \text { on }(0, \delta] .
\end{gathered}
$$

The proofs of (4.2) and (4.3) are as follows. Recall that the condition i) implies (3.2) and so

$$
\left(\frac{t}{\delta}\right)^{\varepsilon} \leq \frac{\mu(t)}{\mu(\delta)} \quad \text { on }(0, \delta]
$$

Since $0<a \varepsilon<h$ is assumed, we have

$$
\left(\frac{t}{\delta}\right)^{h} \leq\left(\frac{t}{\delta}\right)^{a \varepsilon} \leq\left(\frac{\mu(t)}{\mu(\delta)}\right)^{a} \quad \text { on }(0, \delta]
$$

which proves (4.2). Moreover, by the integration by parts and using the condition i) we have

$$
\begin{aligned}
\int_{t}^{\delta} \frac{1}{\tau^{h+1}} \mu(\tau)^{a} d \tau & =\left[\frac{-1}{h} \frac{1}{\tau^{h}} \mu(\tau)^{a}\right]_{t}^{\delta}+\frac{a}{h} \int_{t}^{\delta} \frac{1}{\tau^{h}} \mu(\tau)^{a-1} \mu_{\tau}^{\prime}(\tau) d \tau \\
& \leq \frac{1}{h} \frac{1}{t^{h}} \mu(t)^{a}+\frac{a}{h} \int_{t}^{\delta} \frac{1}{\tau^{h+1}} \mu(\tau)^{a-1}(\varepsilon \mu(\tau)) d \tau \\
& =\frac{1}{h} \frac{1}{t^{h}} \mu(t)^{a}+\frac{a \varepsilon}{h} \int_{t}^{\delta} \frac{1}{\tau^{h+1}} \mu(\tau)^{a} d \tau
\end{aligned}
$$

and therefore we obtain

$$
\int_{t}^{\delta} \frac{1}{\tau^{h+1}} \mu(\tau)^{a} d \tau \leq \frac{1}{h-a \varepsilon} \frac{1}{t^{h}} \mu(t)^{a} \quad \text { on }(0, \delta]
$$

which leads us to (4.3).

Next let us consider

$$
C\left(t \frac{\partial}{\partial t}, x\right) u=f
$$

Since $\lambda_{1}(x), \ldots, \lambda_{m}(x)$ are solutions of $C(\lambda, x)=0$ in $\lambda$, the equation [4.4) is written as 


$$
\left(t \frac{\partial}{\partial t}-\lambda_{1}(x)\right) \cdots\left(t \frac{\partial}{\partial t}-\lambda_{m}(x)\right) u=f .
$$

Therefore, applying Lemma $2 m$-times to this equation we obtain

LEMma 3. Assume the condition (3.4), and assume that $u, f \in \tilde{\mathcal{O}}_{+}(\mu(t))$ satisfy the equation (4.4). Then, if $f \in \tilde{\mathcal{O}}_{a}(\mu(t))$ holds for some $a>0$ we have $u \in \tilde{\mathcal{O}}_{a}(\mu(t))$.

Denote

$$
R[u]=F\left(t, x,\left\{\left(t \frac{\partial}{\partial t}\right)^{j}\left(\frac{\partial}{\partial x}\right)^{\alpha} u\right\}_{\substack{j+|\alpha| \leq m \\ j<m}}\right)-\sum_{j<m} \frac{\partial F}{\partial Z_{j, 0}}(0, x, 0)\left(t \frac{\partial}{\partial t}\right)^{j} u .
$$

The equation $(\mathrm{E})$ is written as

$$
C\left(t \frac{\partial}{\partial t}, x\right) u=R[u]
$$

Moreover we have

LEMMA 4. If $u \in \tilde{\mathcal{O}}_{a}(\mu(t))$ holds for some $a>0$ we have $R[u] \in \tilde{\mathcal{O}}_{b}(\mu(t))$ for any $b$ with $0<b \leq \min \{2 a, m\}$.

Proof. By [5, Lemma 11] we know that

$$
\mu(t+c t)=O(\mu(t)) \quad(\text { as } t \rightarrow+0)
$$

for some $c>0$ and hence we can see that $u \in \tilde{\mathcal{O}}_{a}(\mu(t))$ implies

$$
\left(t \frac{\partial}{\partial t}\right)^{j}\left(\frac{\partial}{\partial x}\right)^{\alpha} u \in \tilde{\mathcal{O}}_{a}(\mu(t)), \quad j+|\alpha| \leq m \text { and } j<m
$$

(see the proof of [5, Theorem 3]).

Therefore, by $\left(\mathrm{A}_{1}\right),\left(\mathrm{A}_{2}\right)$ and $\left(\mathrm{A}_{3}\right)$ we have

$$
\begin{aligned}
R[u]= & F(t, x, 0) \\
& +\sum_{j<m}\left(\frac{\partial F}{\partial Z_{j, 0}}(t, x, 0)-\frac{\partial F}{\partial Z_{j, 0}}(0, x, 0)\right)\left(t \frac{\partial}{\partial t}\right)^{j} u \\
& +\sum_{\substack{j+|\alpha| \leq m \\
|\alpha|>0}} \frac{\partial F}{\partial Z_{j, \alpha}}(t, x, 0)\left(t \frac{\partial}{\partial t}\right)^{j}\left(\frac{\partial}{\partial x}\right)^{\alpha} u \\
& +\sum_{j+|\alpha| \leq m} \sum_{\substack{k+|\beta| \leq m \\
k<m}} O\left(\left(t \frac{\partial}{\partial t}\right)^{j}\left(\frac{\partial}{\partial x}\right)^{\alpha} u \times\left(t \frac{\partial}{\partial t}\right)^{k}\left(\frac{\partial}{\partial x}\right)^{\beta} u\right) \\
= & O(|t|)+O(|t|) O\left(\mu(|t|)^{a}\right)+O\left(O\left(\mu(|t|)^{a}\right) \times O\left(\mu(|t|)^{a}\right)\right) .
\end{aligned}
$$


Since $|t|=O\left(\mu(|t|)^{m}\right) \quad$ (as $t \rightarrow+0$ ) is already proved in (3.2) with $\varepsilon=1 / m$, we obtain the conclusion of Lemma 4 .

Now, by using Lemmas 3 and 4 let us prove Proposition 1 .

Proof of Proposition 1. Let $u \in \tilde{\mathcal{O}}_{+}(\mu(t))$ be a solution of $(\mathrm{E})$. Then, by the definition of $\tilde{\mathcal{O}}_{+}(\mu(t))$ we have $u \in \tilde{\mathcal{O}}_{a}(\mu(t))$ for some $a>0$. Choose a sequence $a_{0}, a_{1}, \ldots, a_{N}$ such that

i) $a_{0}=a<a_{1}<a_{2}<\cdots<a_{N}=m$, and

ii) $a_{i+1} \leq \min \left\{2 a_{i}, m\right\}$ for $i=0,1, \ldots, N-1$.

Since $u \in \tilde{\mathcal{O}}_{a_{0}}(\mu(t))$ is known, by Lemma 4 we have $R[u] \in \tilde{\mathcal{O}}_{a_{1}}(\mu(t))$ and therefore by applying Lemma 3 to the equation $C(t \partial / \partial t, x) u=R[u]$ we have $u \in \tilde{\mathcal{O}}_{a_{1}}(\mu(t))$. Then, by Lemma 4 we have $R[u] \in \tilde{\mathcal{O}}_{a_{2}}(\mu(t))$ and so applying Lemma 3 again to $C(t \partial / \partial t, x) u=R[u] \in \tilde{\mathcal{O}}_{a_{2}}(\mu(t))$ we have $u \in \tilde{\mathcal{O}}_{a_{2}}(\mu(t))$.

Thus, by repeating the same argument as above we obtain $u \in \tilde{\mathscr{O}}_{a_{N}}(\mu(t))$. Since $a_{N}=m$, this completes the proof of Proposition 1 .

\section{§5. Proof of $\mathbf{C}_{2}$ ).}

The assertion $\mathrm{C}_{2}$ ) comes from the following proposition.

Proposition 2. Let $\mu(t)$ be a weight function satisfying

$$
\mu(t) \in C^{1}((0, T)) \quad \text { and } \quad\left(t \frac{d \mu}{d t}\right)(t)=O(\mu(t)) \quad(\text { as } t \rightarrow+0) .
$$

Assume the condition (3.4). Then, if $u \in \tilde{\mathcal{O}}_{m}(\mu(t))$ is a solution of $(\mathrm{E})$ we have $u \in \tilde{\mathcal{O}}_{+}$.

We will prove this proposition from now. By (5.1) we have

$$
t \mu_{t}^{\prime}(t) \leq A \mu(t) \quad \text { on }(0, T)
$$

for some $A>0$. Also, by (3.4) we can find $h>0$ and $R>0$ such that

$$
\operatorname{Re} \lambda_{i}(x) \geq 2 h>0 \quad \text { on } D_{R}, \quad i=1, \ldots, m .
$$

Without loss of generality we may assume that $0<h<1$ holds.

Let $u \in \tilde{\mathcal{O}}_{m}(\mu(t))$ be a solution of (E), and assume that $u(t, x)$ is holomorphic on $S(\varepsilon(s)) \times D_{2 R}$ where $\varepsilon(s)$ is a positive-valued continuous function and $R>0$ is sufficiently small. Since the condition (5.1) is assumed, by [5, Lemma 11] we have $\mu(t+c t)=O(\mu(t))$ (as $t \rightarrow+0)$ for some $c>0$ and by the same argument as in the proof of [5, Theorem 3] we have

$$
\left(t \frac{\partial}{\partial t}\right)^{j}\left(\frac{\partial}{\partial x}\right)^{\alpha} u \in \tilde{\mathcal{O}}_{m}(\mu(t)) \text { for } j+|\alpha| \leq m \text { and } j<m .
$$

Therefore, for any $\theta_{0}>0$ we can find $\delta>0$ and $M>0$ such that 


$$
\begin{gathered}
\left|\left(t \frac{\partial}{\partial t}\right)^{j}\left(\frac{\partial}{\partial x}\right)^{\alpha} u(t, x)\right| \leq M \mu(|t|)^{m} \text { on } S_{\theta_{0}}(\delta) \times D_{R} \\
\text { for } j+|\alpha| \leq m \text { and } j<m
\end{gathered}
$$

where $S_{\theta_{0}}(\delta)=\left\{t \in S_{\theta_{0}} ; 0<|t| \leq \delta\right\}$.

Our purpose is to show the following: if $R_{1}>0$ is sufficiently small, for any $\theta_{0}>0$ we can find $\delta_{1}>0$ and $M_{1}>0$ such that

$$
|u(t, x)| \leq M_{1}|t|^{h} \quad \text { on } S_{\theta_{0}}\left(\delta_{1}\right) \times D_{R_{1}} .
$$

The rest part of this section is used to prove this estimate.

Denote

$$
\begin{aligned}
\Theta_{0} & =1 \\
\Theta_{1} & =\left(t \frac{\partial}{\partial t}-\lambda_{1}(0)\right) \\
\Theta_{2} & =\left(t \frac{\partial}{\partial t}-\lambda_{2}(0)\right)\left(t \frac{\partial}{\partial t}-\lambda_{1}(0)\right) \\
& \ldots \ldots \ldots \ldots \\
& \ldots \ldots \ldots \ldots \\
& \ldots \ldots \ldots \ldots \\
\Theta_{m} & =\left(t \frac{\partial}{\partial t}-\lambda_{m}(0)\right)\left(t \frac{\partial}{\partial t}-\lambda_{m-1}(0)\right) \cdots\left(t \frac{\partial}{\partial t}-\lambda_{1}(0)\right) .
\end{aligned}
$$

Since $u \in \tilde{\mathcal{O}}_{m}(\mu(t))$ is a solution of $(\mathrm{E})$, we have

$$
\begin{aligned}
\Theta_{m} u= & F(t, x, 0) \\
& +\sum_{j<m}\left(\frac{\partial F}{\partial Z_{j, 0}}(t, x, 0)-\frac{\partial F}{\partial Z_{j, 0}}(0,0,0)\right)\left(t \frac{\partial}{\partial t}\right)^{j} u \\
& +\sum_{j+|\alpha| \leq m} \frac{\partial F}{\partial Z_{j, \alpha} \mid>0}(t, x, 0)\left(t \frac{\partial}{\partial t}\right)^{j}\left(\frac{\partial}{\partial x}\right)^{\alpha} u \\
& +\sum_{\substack{j+|\alpha| \leq m \\
j<m}} \sum_{\substack{k+|\beta| \leq m \\
k<m}} O\left(\left(t \frac{\partial}{\partial t}\right)^{j}\left(\frac{\partial}{\partial x}\right)^{\alpha} u \times\left(t \frac{\partial}{\partial t}\right)^{k}\left(\frac{\partial}{\partial x}\right)^{\beta} u\right) \\
= & F(t, x, 0)+\sum_{j<m} a_{j}(t, x) \Theta_{j} u+\sum_{\substack{j+|\alpha| \leq m \\
j<m}} b_{j, \alpha}(t, x) \Theta_{j}\left(\frac{\partial}{\partial x}\right)^{\alpha} u,
\end{aligned}
$$


where $a_{j}(t, x)(j<m)$ are holomorphic functions in a neighborhood of $(0,0)$ satisfying $a_{j}(0,0)=0$, and $b_{j, \alpha}(t, x) \quad(j+|\alpha| \leq m, j<m)$ are functions in $\tilde{\mathcal{O}}_{m}(\mu(t))$. Note that $a_{j}(t, x) \quad(j<m)$ are independent of $u$, but $b_{j, \alpha}(t, x) \quad(j+|\alpha| \leq m, j<m)$ depend on $u$.

Introduce the following notation. For a formal power series $f(t, x)$ in $x$ with coefficients in $C^{0}((0, T))$ of the form

$$
f(t, x)=\sum_{\alpha \in N^{n}} f_{\alpha}(t) x^{\alpha}, \quad f_{\alpha}(t) \in C^{0}((0, T))
$$

we write

$$
\|f(t)\|_{\rho}=\sum_{\alpha \in N^{n}}\left|f_{\alpha}(t)\right| \frac{\alpha !}{|\alpha| !} \rho^{|\alpha|}
$$

(which is a formal power series in $\rho$ with coefficients in $C^{0}((0, T))$ ). In case $f(t, x)$ is a function on $(0, T) \times D_{R}$ continuous in $t$ and holomorphic in $x$, by using the Taylor expansion of $f(t, x)$ in $x$ we can define $\|f(t)\|_{\rho}$ in the same way. Note that the following majorant relation holds:

$$
\left\|\left(\frac{\partial}{\partial x_{i}}\right) f(t)\right\|_{\rho} \ll \frac{\partial}{\partial \rho}\|f(t)\|_{\rho}, \quad i=1, \ldots, n .
$$

Take any $\theta_{0}>0$. Let $R>0$ and $\delta>0$ be the ones in (5.4). Note that $\delta$ depends on $\theta_{0}$ but $R$ is independent of $\theta_{0}$. For $(j, k) \in \boldsymbol{N} \times \boldsymbol{N}$ satisfying $j+k \leq$ $m-1$ we set

$$
\begin{array}{r}
\psi_{j, k}(t, \rho, \theta)=\mu(t)^{k} \times \sum_{|\alpha|=k}\left\|\Theta_{j}\left(\frac{\partial}{\partial x}\right)^{\alpha} u\left(t e^{\sqrt{-1} \theta}\right)\right\|_{\rho} \\
\phi_{j, k}(t, \rho, \theta)=\int_{t}^{\delta}\left(\frac{t}{\tau}\right)^{\operatorname{Re} \lambda_{j+1}(0)} \mu(\tau)^{k} \\
\times\left\{\sum_{|\alpha|=k}\left\|\Theta_{j+1}\left(\frac{\partial}{\partial x}\right)^{\alpha} u\left(\tau e^{\sqrt{-1} \theta}\right)\right\|_{\rho}\right. \\
\left.+k A \sum_{|\alpha|=k}\left\|\Theta_{j}\left(\frac{\partial}{\partial x}\right)^{\alpha} u\left(\tau e^{\sqrt{-1} \theta}\right)\right\|_{\rho}\right\} \frac{d \tau}{\tau} .
\end{array}
$$

Then, by the argument similar to the proof of [4, Lemma 3] we have 
Lemma 5. $\quad \psi_{j, k}(t, \rho, \theta)(j+k \leq m-1)$ and $\phi_{j, k}(t, \rho, \theta)(j+k \leq m-1)$ are well-defined in $C^{0}\left([0, \delta] \times[0, R] \times\left(-\theta_{0}, \theta_{0}\right)\right)$ and satisfy the following properties (1) (4) on $\left\{(t, \rho, \theta) ; 0<t \leq \delta, 0 \leq \rho \leq R\right.$ and $\left.|\theta|<\theta_{0}\right\}$ :

(1) For any $(j, k)$ we have

$$
\psi_{j, k}(t, \rho, \theta) \leq\left(\frac{t}{\delta}\right)^{2 h} \psi_{j, k}(\delta, \rho, \theta)+\phi_{j, k}(t, \rho, \theta)
$$

(2) When $k>0$, we have

$$
\begin{aligned}
& \left(-t \frac{\partial}{\partial t}+2 h\right) \phi_{j, k}(t, \rho, \theta) \\
& \quad \leq n \mu(t) \frac{\partial}{\partial \rho} \psi_{j+1, k-1}(t, \rho, \theta)+n k A \mu(t) \frac{\partial}{\partial \rho} \psi_{j, k-1}(t, \rho, \theta) .
\end{aligned}
$$

(3) When $k=0$ and $j=0,1, \ldots, m-2$, we have

$$
\left(-t \frac{\partial}{\partial t}+2 h\right) \phi_{j, 0}(t, \rho, \theta) \leq \psi_{j+1,0}(t, \rho, \theta)
$$

(4) When $k=0$ and $j=m-1$, we have

$$
\begin{aligned}
\left(-t \frac{\partial}{\partial t}+2 h\right) \phi_{m-1,0}(t, \rho, \theta) \\
\leq K t+(a(t, \rho)+b(t, \rho)) \sum_{j<m} \psi_{j, 0}(t, \rho, \theta) \\
\quad+B \mu(t) \frac{\partial}{\partial \rho} \sum_{j+k \leq m-1} \psi_{j, k}(t, \rho, \theta)
\end{aligned}
$$

for some $K>0, B>0, a(t, \rho) \in C^{0}([0, \delta] \times[0, R])$ with $a(0,0)=0$, and $b(t, \rho) \in$ $C^{0}([0, \delta] \times[0, R])$ with $b(t, \rho)=O\left(\mu(t)^{m}\right) \quad($ as $t \rightarrow+0$ uniformly in $\rho \in[0, R])$. Moreover, by (5.6) we see that $K$ and $a(t, \rho)$ are independent of $\theta_{0}$.

Proof. Set

$$
u_{j, k}(t, x)=\mu(t)^{k} \Theta_{j}\left(\frac{\partial}{\partial x}\right)^{\alpha} u\left(t e^{\sqrt{-1} \theta}, x\right)
$$

Then we have 


$$
\begin{aligned}
\left(t \frac{\partial}{\partial t}\right. & \left.-\lambda_{j+1}(0)\right) u_{j, k}(t, x) \\
& =\mu(t)^{k} \Theta_{j+1}\left(\frac{\partial}{\partial x}\right)^{\alpha} u\left(t e^{\sqrt{-1} \theta}, x\right)+k t \mu_{t}^{\prime}(t) \mu(t)^{k-1} \Theta_{j}\left(\frac{\partial}{\partial x}\right)^{\alpha} u\left(t e^{\sqrt{-1} \theta}, x\right)
\end{aligned}
$$

and by integrating this from $t$ to $\delta$ we have

$$
\begin{aligned}
u_{j, k}(t, x)=\left(\frac{t}{\delta}\right)^{\lambda_{j+1}(0)} u_{j, k}(\delta, x) & \\
-\int_{t}^{\delta}\left(\frac{t}{\tau}\right)^{\lambda_{j+1}(0)} & \left\{\mu(\tau)^{k} \Theta_{j+1}\left(\frac{\partial}{\partial x}\right)^{\alpha} u\left(\tau e^{\sqrt{-1} \theta}, x\right)\right. \\
& \left.\quad+k \tau \mu_{\tau}^{\prime}(\tau) \mu(\tau)^{k-1} \Theta_{j}\left(\frac{\partial}{\partial x}\right)^{\alpha} u\left(\tau e^{\sqrt{-1} \theta}, x\right)\right\} \frac{d \tau}{\tau} .
\end{aligned}
$$

Therefore by taking the norm and by using (5.2) and (5.3) we obtain

$$
\begin{gathered}
\mu(t)^{k}\left\|\Theta_{j}\left(\frac{\partial}{\partial x}\right)^{\alpha} u\left(t e^{\sqrt{-1} \theta}\right)\right\|_{\rho}=\left\|u_{j, k}(t)\right\|_{\rho} \\
\leq\left(\frac{t}{\delta}\right)^{\operatorname{Re} \lambda_{j+1}(0)}\left\|u_{j, k}(\delta)\right\|_{\rho} \\
\quad+\int_{t}^{\delta}\left(\frac{t}{\tau}\right)^{\operatorname{Re} \lambda_{j+1}(0)}\left\{\mu(\tau)^{k}\left\|\Theta_{j+1}\left(\frac{\partial}{\partial x}\right)^{\alpha} u\left(\tau e^{\sqrt{-1} \theta}\right)\right\|_{\rho}\right. \\
\left.\quad+k A \mu(\tau) \mu(\tau)^{k-1}\left\|\Theta_{j}\left(\frac{\partial}{\partial x}\right)^{\alpha} u\left(\tau e^{\sqrt{-1} \theta}\right)\right\|_{\rho}\right\} \frac{d \tau}{\tau} \\
\leq\left(\frac{t}{\delta}\right)^{2 h} \mu(\delta)^{k}\left\|\Theta_{j}\left(\frac{\partial}{\partial x}\right)^{\alpha} u\left(\delta e^{\sqrt{-1} \theta}\right)\right\|_{\rho} \\
+\int_{t}^{\delta}\left(\frac{t}{\tau}\right)^{\operatorname{Re} \lambda_{j+1}(0)} \mu(\tau)^{k}\left\{\left\|\Theta_{j+1}\left(\frac{\partial}{\partial x}\right)^{\alpha} u\left(\tau e^{\sqrt{-1} \theta}\right)\right\|_{\rho}\right. \\
\left.+k A\left\|\Theta_{j}\left(\frac{\partial}{\partial x}\right)^{\alpha} u\left(\tau e^{\sqrt{-1} \theta}\right)\right\|_{\rho}\right\} \frac{d \tau}{\tau}
\end{gathered}
$$

which leads us to the property (1).

Denote: $e_{1}=(1,0, \ldots, 0), \ldots, e_{n}=(0, \ldots, 0,1) \in \boldsymbol{N}^{n}$. If $|\alpha|>0$ we have

$$
\left(\frac{\partial}{\partial x}\right)^{\alpha}=\left(\frac{\partial}{\partial x_{i}}\right)\left(\frac{\partial}{\partial x}\right)^{\alpha-e_{i}}
$$


for some $i=i_{\alpha}$ and

$$
\left\|\Theta_{l}\left(\frac{\partial}{\partial x}\right)^{\alpha} u\left(t e^{\sqrt{-1} \theta}\right)\right\|_{\rho} \leq \frac{\partial}{\partial \rho}\left\|\Theta_{l}\left(\frac{\partial}{\partial x}\right)^{\alpha-e_{i}} u\left(t e^{\sqrt{-1} \theta}\right)\right\|_{\rho}
$$

for any $l=0,1, \ldots, m$ and any $\rho \in[0, R]$.

When $k>0$, by using (5.3) and (5.9) we can verify the property (2) as follows:

$$
\begin{aligned}
& \left(-t \frac{\partial}{\partial t}+2 h\right) \phi_{j, k}(t, \rho, \theta) \\
& \leq\left(-t \frac{\partial}{\partial t}+\operatorname{Re} \lambda_{j+1}(0)\right) \phi_{j, k}(t, \rho, \theta) \\
& =\mu(t)^{k}\left\{\sum_{|\alpha|=k}\left\|\Theta_{j+1}\left(\frac{\partial}{\partial x}\right)^{\alpha} u\left(t e^{\sqrt{-1} \theta}\right)\right\|_{\rho}\right. \\
& \left.\quad+k A \sum_{|\alpha|=k}\left\|\Theta_{j}\left(\frac{\partial}{\partial x}\right)^{\alpha} u\left(t e^{\sqrt{-1} \theta}\right)\right\|_{\rho}\right\} \\
& \leq \mu(t)^{k}\left\{\sum_{|\alpha|=k} \frac{\partial}{\partial \rho}\left\|\Theta_{j+1}\left(\frac{\partial}{\partial x}\right)^{\alpha-e_{i}} u\left(t e^{\sqrt{-1} \theta}\right)\right\|_{\rho}\right. \\
& \left.\quad+k A \sum_{|\alpha|=k} \frac{\partial}{\partial \rho}\left\|\Theta_{j}\left(\frac{\partial}{\partial x}\right)^{\alpha-e_{i}} u\left(t e^{\sqrt{-1} \theta}\right)\right\|_{\rho}\right\} \\
& \leq n \mu(t) \frac{\partial}{\partial \rho} \psi_{j+1, k-1}(t, \rho, \theta)+n k A \mu(t) \frac{\partial}{\partial \rho} \psi_{j, k-1}(t, \rho, \theta) .
\end{aligned}
$$

When $k=0$ and $j=0,1, \ldots, m-2$, the property (3) is verified by:

$$
\begin{aligned}
\left(-t \frac{\partial}{\partial t}+2 h\right) \phi_{j, 0}(t, \rho, \theta) & \leq\left(-t \frac{\partial}{\partial t}+\operatorname{Re} \lambda_{j+1}(0)\right) \phi_{j, 0}(t, \rho, \theta) \\
& =\left\|\Theta_{j+1} u\left(t e^{\sqrt{-1} \theta}\right)\right\|_{\rho}=\psi_{j+1,0}(t, \rho, \theta) .
\end{aligned}
$$

When $k=0$ and $j=m-1$ we have

$$
\begin{aligned}
& \left(-t \frac{\partial}{\partial t}+2 h\right) \phi_{m-1,0}(t, \rho, \theta) \\
& \quad \leq\left(-t \frac{\partial}{\partial t}+\operatorname{Re} \lambda_{m}(0)\right) \phi_{m-1,0}(t, \rho, \theta)=\left\|\Theta_{m} u\left(t e^{\sqrt{-1} \theta}\right)\right\|_{\rho} .
\end{aligned}
$$


On the other hand, by (5.6) we know that the equation (E) is written as

$$
\begin{aligned}
\Theta_{m} u= & F(t, x, 0)+\sum_{j<m}\left(a_{j}(t, x)+b_{j, 0}(t, x)\right) \Theta_{j} u \\
& +\sum_{\substack{j+|\alpha| \leq m \\
|\alpha|>0}} b_{j, \alpha}(t, x) \Theta_{j}\left(\frac{\partial}{\partial x}\right)^{\alpha} u \\
= & O(|t|)+\sum_{j<m}\left(O(|t|+|x|)+O\left(\mu(|t|)^{m}\right)\right) \Theta_{j} u \\
& +\sum_{\substack{j+|\alpha| \leq m \\
|\alpha|>0}} O\left(\mu(|t|)^{m}\right) \Theta_{j}\left(\frac{\partial}{\partial x}\right)^{\alpha} u .
\end{aligned}
$$

Therefore, by taking the norm and by using [5.9] we have

$$
\begin{aligned}
\left\|\Theta_{m} u\left(t e^{\sqrt{-1} \theta}\right)\right\|_{\rho} \\
\leq K t+\left(O(t+\rho)+O\left(\mu(t)^{m}\right)\right) \sum_{j<m}\left\|\Theta_{j} u\left(t e^{\sqrt{-1} \theta}\right)\right\|_{\rho} \\
\quad+\sum_{\substack{j+|\alpha| \leq m \\
|\alpha|>0}} O\left(\mu(t)^{m}\right) \frac{\partial}{\partial \rho}\left\|\Theta_{j}\left(\frac{\partial}{\partial x}\right)^{\alpha-e_{i}} u\left(t e^{\sqrt{-1} \theta}\right)\right\|_{\rho} \\
\leq K t+\left(O(t+\rho)+O\left(\mu(t)^{m}\right)\right) \sum_{j<m} \psi_{j, 0}(t, \rho, \theta) \\
\quad+O(\mu(t)) \frac{\partial}{\partial \rho_{j+k \leq m-1}} \sum_{j, k}(t, \rho, \theta) .
\end{aligned}
$$

Hence, combining this with (5.10) we obtain the property (4).

Next, we choose $\sigma_{j}>0(j=0,1, \ldots, m-1)$ so that

$$
\frac{\sigma_{j}}{\sigma_{j+1}}<\frac{h}{2}, \quad j=0,1, \ldots, m-2
$$

hold and then we choose $\delta_{2}>0$ and $R_{2}>0$ sufficiently small so that

$$
\frac{\sigma_{m-1}}{\sigma_{j}} a(t, \rho)<\frac{h}{4}, \quad j=0,1, \ldots, m-1,
$$




$$
\frac{\sigma_{m-1}}{\sigma_{j}} b(t, \rho)<\frac{h}{4}, \quad j=0,1, \ldots, m-1
$$

hold on $\left\{(t, \rho) ; 0 \leq t \leq \delta_{2}, 0 \leq \rho \leq R_{2}\right\}$. Since $a(t, \rho)$ is independent of $\theta_{0}$ we may assume that $R_{2}>0$ is also independent of $\theta_{0}$.

Set

$$
\begin{aligned}
& \Psi(t, \rho, \theta)=\sum_{j+k \leq m-1} \psi_{j, k}(t, \rho, \theta), \\
& \Phi(t, \rho, \theta)=\sum_{j<m} \sigma_{j} \phi_{j, 0}(t, \rho, \theta)+\sum_{\substack{j+k \leq m-1 \\
k>0}} \phi_{j, k}(t, \rho, \theta) .
\end{aligned}
$$

Then we have:

Lemma 6. There are $C_{1}>0$ and $C_{2}>0$ such that

$$
\begin{aligned}
\left(-t \frac{\partial}{\partial t}+h\right) \Phi(t, \rho, \theta) & \\
\leq & \sigma_{m-1} K t+C_{1}\left(\frac{t}{\delta}\right)^{2 h}\left(1+\mu(t) \frac{\partial}{\partial \rho}\right) \Psi(\delta, \rho, \theta) \\
& +C_{2} \mu(t) \frac{\partial}{\partial \rho} \Phi(t, \rho, \theta)
\end{aligned}
$$

holds on $\left\{(t, \rho, \theta) ; 0<t \leq \delta_{2}, 0 \leq \rho \leq R_{2}\right.$ and $\left.|\theta|<\theta_{0}\right\}$.

Proof. By using (2) (4) of Lemma 5 we have

$$
\begin{aligned}
\left(-t \frac{\partial}{\partial t}+\right. & 2 h) \Phi(t, \rho, \theta) \\
\leq & \sum_{j \leq m-2} \sigma_{j} \psi_{j+1,0}(t, \rho, \theta) \\
& +\sigma_{m-1} K t+\sigma_{m-1}(a(t, \rho)+b(t, \rho)) \sum_{j<m} \psi_{j, 0}(t, \rho, \theta) \\
& +C_{3} \mu(t) \frac{\partial}{\partial \rho} \sum_{j+k \leq m-1} \psi_{j, k}(t, \rho, \theta)
\end{aligned}
$$

for some $C_{3}>0$, and therefore by (1) of Lemma 5, (5.11), (5.12) and (5.13) we obtain 


$$
\begin{aligned}
\left(-t \frac{\partial}{\partial t}+2 h\right) \Phi(t, \rho, \theta) \\
\leq \sum_{j \leq m-2} \frac{h}{2} \sigma_{j+1}\left[\left(\frac{t}{\delta}\right)^{2 h} \psi_{j+1,0}(\delta, \rho, \theta)+\phi_{j+1,0}(t, \rho, \theta)\right]+\sigma_{m-1} K t \\
+\sum_{j<m}\left(\frac{h}{4}+\frac{h}{4}\right) \sigma_{j}\left[\left(\frac{t}{\delta}\right)^{2 h} \psi_{j, 0}(\delta, \rho, \theta)+\phi_{j, 0}(t, \rho, \theta)\right] \\
+C_{3} \mu(t) \frac{\partial}{\partial \rho} \sum_{j+k \leq m-1}\left[\left(\frac{t}{\delta}\right)^{2 h} \psi_{j, k}(\delta, \rho, \theta)+\phi_{j, k}(t, \rho, \theta)\right] \\
\leq\left(\frac{h}{2}+\frac{h}{4}+\frac{h}{4}\right) \Phi(t, \rho, \theta)+\sigma_{m-1} K t \\
\quad+C_{1}\left(\frac{t}{\delta}\right)^{2 h}\left(1+\mu(t) \frac{\partial}{\partial \rho}\right) \Psi(\delta, \rho, \theta)+C_{2} \mu(t) \frac{\partial}{\partial \rho} \Phi(t, \rho, \theta)
\end{aligned}
$$

for some $C_{1}>0$ and $C_{2}>0$. This immediately leads us to (5.14).

Now, let us complete the proof of Proposition 2. Set

$$
M_{2}=\sigma_{m-1} K+\frac{C_{1}}{\delta^{2 h}} \sup _{\substack{0 \leq \rho \leq R_{2} \\|\theta|<\theta_{0}}}\left(\left(1+\mu\left(\delta_{2}\right) \frac{\partial}{\partial \rho}\right) \Psi(\delta, \rho, \theta)\right)
$$

Then, by Lemma 6 we have

$$
\left(-t \frac{\partial}{\partial t}+h-C_{2} \mu(t) \frac{\partial}{\partial \rho}\right) \Phi(t, \rho, \theta) \leq M_{2}\left(t+t^{2 h}\right)
$$

on $\left\{(t, \rho, \theta) ; 0<t \leq \delta_{2}, 0 \leq \rho \leq R_{2}\right.$ and $\left.|\theta|<\theta_{0}\right\}$.

Completion of THE PRoOF of Proposition 2. Take any $R_{1}$ such that $0<$ $R_{1}<R_{2}$, and then choose $\delta_{1}>0$ so that $0<\delta_{1}<\delta_{2}$ and

$$
R_{1}+C_{2} \int_{0}^{\delta_{1}} \frac{\mu(s)}{s} d s \leq R_{2}
$$

Define the function $\rho(t)$ by

$$
\rho(t)=R_{1}+C_{2} \int_{0}^{t} \frac{\mu(s)}{s} d s \quad \text { for } 0 \leq t \leq \delta_{1}
$$


Then, $R_{1} \leq \rho(t) \leq R_{2}$ for $0 \leq t \leq \delta_{1}, t(d \rho / d t)=C_{2} \mu(t)$, and $\rho(t)$ is increasing in $t$. Moreover we have

$$
\left[0, \delta_{1}\right] \times\left[0, R_{1}\right] \subset\left\{(t, \rho) ; 0 \leq t \leq \delta_{1}, 0 \leq \rho \leq \rho(t)\right\} .
$$

Set

$$
\varphi(t, \theta)=\Phi(t, \rho(t), \theta) \quad \text { for } 0 \leq t \leq \delta_{1} \text { and }|\theta|<\theta_{0} .
$$

By (5.15) we have

$$
\begin{aligned}
\left(-t \frac{\partial}{\partial t}+h\right) \varphi(t, \theta) & =\left(-t \frac{\partial}{\partial t}+h\right) \Phi-\frac{\partial \Phi}{\partial \rho} t \frac{d \rho(t)}{d t} \\
& =\left(-t \frac{\partial}{\partial t}+h-C_{2} \mu(t) \frac{\partial}{\partial \rho}\right) \Phi \\
& \leq M_{2}\left(t+t^{2 h}\right),
\end{aligned}
$$

that is

$$
\left(-t \frac{\partial}{\partial t}+h\right) \varphi(t, \theta) \leq M_{2}\left(t+t^{2 h}\right), \quad 0<t \leq \delta_{1} \text { and }|\theta|<\theta_{0}
$$

which is equivalent to

$$
-\frac{\partial}{\partial t}\left(t^{-h} \varphi(t, \theta)\right) \leq M_{2}\left(\frac{1}{t^{h}}+\frac{1}{t^{1-h}}\right), \quad 0<t \leq \delta_{1} \text { and }|\theta|<\theta_{0} .
$$

Since $0<h<1$ is assumed, by integrating this from $t$ to $\delta_{1}$ we have

$$
t^{-h} \varphi(t, \theta) \leq \delta_{1}^{-h} \varphi\left(\delta_{1}, \theta\right)+M_{2}\left(\frac{\delta_{1}^{1-h}}{1-h}+\frac{\delta_{1}^{h}}{h}\right)
$$

and hence

$$
\varphi(t, \theta) \leq M_{3} t^{h}, \quad 0<t \leq \delta_{1} \text { and }|\theta|<\theta_{0}
$$

where

$$
M_{3}=\frac{1}{\delta_{1}^{h}} \sup _{\substack{0 \leq \rho \leq R_{2} \\|\theta|<\theta_{0}}}\left(\Phi\left(\delta_{1}, \rho, \theta\right)\right)+M_{2}\left(\frac{\delta_{1}^{1-h}}{1-h}+\frac{\delta_{1}^{h}}{h}\right) .
$$

Thus, if we notice the fact that $\Phi(t, \rho, \theta)$ is increasing in $\rho$, by (5.16), (5.17) and (5.18) we obtain

$$
\Phi(t, \rho, \theta) \leq M_{3} t^{h}
$$


on $\left\{(t, \rho, \theta) ; 0<t \leq \delta_{1}, 0 \leq \rho \leq R_{1}\right.$ and $\left.|\theta|<\theta_{0}\right\}$.

Finally, let us show that the estimate (5.5) follows from (5.19). Note that

$$
\psi_{0,0}(t, \rho, \theta)=\left\|u\left(t e^{\sqrt{-1} \theta}\right)\right\|_{\rho}
$$

holds. Therefore, by (5.19) and (1) of Lemma 5 we have

$$
\begin{aligned}
\left\|u\left(t e^{\sqrt{-1} \theta}\right)\right\|_{\rho} & \leq\left(\frac{t}{\delta}\right)^{2 h}\left\|u\left(\delta e^{\sqrt{-1} \theta}\right)\right\|_{\rho}+\phi_{0,0}(t, \rho, \theta) \\
& \leq\left(\frac{t}{\delta}\right)^{2 h} \sup _{\substack{0 \leq \rho \leq R_{1} \\
|\theta|<\theta_{0}}}\left\|u\left(\delta e^{\sqrt{-1} \theta}\right)\right\|_{\rho}+\frac{M_{3}}{\sigma_{0}} t^{h}
\end{aligned}
$$

on $\left\{(t, \rho, \theta) ; 0<t \leq \delta_{1}, 0 \leq \rho \leq R_{1}\right.$ and $\left.|\theta|<\theta_{0}\right\}$. This implies (5.5).

Since $R_{1}>0$ is chosen independently of $\theta_{0}$, this completes the proof of Proposition 2.

\section{References}

[1] R. Gérard and H. Tahara, Solutions holomorphes et singulières d'équations aux dérivées partielles singulières non linéaires, Publ. RIMS, Kyoto Univ., 29 (1993), 121-151.

[2] R. Gérard and H. Tahara, Singular nonlinear partial differential equations, Aspects of Mathematics, E 28, Vieweg, 1996.

[3] H. Tahara, Removable singularities of solutions of nonlinear singular partial differential equations, Banach Center Publications, 33 (1996), 395-399.

[ 4 ] H. Tahara, Uniqueness of the solution of non-linear singular partial differential equations, J. Math. Soc. Japan, 48 (1996), 729-744.

[ 5 ] H. Tahara, On the uniqueness theorem for nonlinear singular partial differential equations, J. Math. Sci. Univ. Tokyo, 5 (1998), 477-506.

Hidetoshi TAHARA

Department of Mathematics

Sophia University

Kioicho, Chiyoda-ku

Tokyo 102-8554

Japan 